\title{
Análisis de la velocidad y la aceleración entre un golpe de boxeo y uno de taekwondo
}

\section{Analysis of speed and acceleration between a boxing attack and taekwondo}

\author{
Diego Alonso Sánchez-Rodríguez ${ }^{1}$; Andrés Felipe Bohórquez-Aldana ${ }^{2}$
}

\begin{abstract}
'Licenciado en Educación Física, M.Sc. Universidad de Ciencias Aplicadas y Ambientales U.D.C.A. Bogotá, D.C., Colombia; e-mail: disanchez@udca.edu.co; (Dhttps://orcid.org/0000-0003-4110-1572

2Profesional en Ciencias del Deporte, M.Sc. Universidad de Ciencias Aplicadas y Ambientales U.D.C.A. Bogotá, D.C., Colombia; e-mail: anbohorquez@udca. edu.co; Dhttps://orcid.org/0000-0002-5983-8188
\end{abstract}

Cómo citar: Sánchez-Rodríguez, D.A.; Bohórquez-Aldana, A.F. 2020. Análisis de la velocidad y la aceleración entre un golpe de boxeo y uno de taekwondo. Rev. U.D.C.A Act. \& Div. Cient. 23(1):e1481. http://doi.org/10.31910/rudca.v23.n1.2020.1481

Artículo de acceso abierto publicado por Revista U.D.C.A Actualidad \& Divulgación Científica, bajo una licencia Creative Commons CC BY-NC 4.0

Publicación oficial de la Universidad de Ciencias Aplicadas y Ambientales U.D.C.A, Institución de educación superior Acreditada de Alta Calidad por el Ministerio de Educación Nacional.

Recibido: Febrero 19 de 2019

Aceptado: Febrero 17 de 2020

Editado por: Ingeborg Zenner de Polanía

\section{RESUMEN}

Dentro del ámbito nacional e internacional, se carecen de estudios que evalúen la semejanza o la diferencia entre los golpes principales de deportes de combate. Por tal razón, la presente investigación tiene como objetivo analizar los golpes más representativos de boxeo y de taekwondo, con los cuales, se pretende reconocer las diferencias entre la velocidad y la aceleración de la ejecución del golpe recto, en boxeo y bandal chagi, en taekwondo. El método utilizado fue observacional, de corte transversal y descriptivo. Mediante captura videográfica, los golpes recto y bandal chagi, fueron analizados por el software Ehuman versión 5.0. El procedimiento estadístico involucró una prueba Mann Whitney U Test, para determinar la semejanza o diferencia entre las variables. La muestra estuvo constituida por 8 boxeadores y 8 taekwondocas, de rama masculina de Bogotá, quienes hacen parte de los equipos de rendimiento de la capital. Dentro de los resultados obtenidos, se destacó la diferencia entre velocidades del golpe recto en boxeo, correspondiente a $4,17 \pm 1,35 \mathrm{~m} / \mathrm{s}$, frente a la patada bandal chagi, de $11,75 \pm 1,04 \mathrm{~m} / \mathrm{s}$; la diferencia estadística fue de $\mathrm{p}=0,0008$. Para la evaluación de aceleración, el golpe recto presentó, como resultado, $12,3 \pm 5,41 \mathrm{~m} / \mathrm{s}^{2}$, mientras que la patada bandal chagi, tuvo un resultado promedio de $74 \pm 37,85 \mathrm{~m} / \mathrm{s}^{2}$; la diferencia estadística fue de $\mathrm{p}=0,00033$, utilizando, para ambas medidas, un nivel de significancia de $\mathrm{p}=0,05$.

Palabras clave: boxeo; Tae kwon Do; golpes de ataque; deporte de combate; aceleración.

\section{ABSTRACT}

Within the national and international scope, studies that assess the similarity or difference between the main knock of combat sports will be seen. For this reason, the maims of this research is to analyze the most representative knock of Boxing and Taekwondo, pretent to recognice the differences between the 
speed and acceleration of the execution of the straight punch in boxing and the chagi bandal in the taekwondo. The method used was observational, cross-sectional and descriptive. It was processed by the video capture of the straight strokes and chagi bandal, it was analyzed by means of the Ehuman software version 5.0. The statistical procedure involved a Mann Whitney U Test to determine the similarity or difference between the variables. The sample was conformed by 8 boxers and 8 takwondo athletes from the male category of Bogotá, who had part of the capital's performance teams. Among the results affected, the difference between speeds of the straight punch in boxing, corresponding to $4.17 \pm 1.35 \mathrm{~m} / \mathrm{s}$, was highlighted, compared to the bandal chagi kick of $11.75 \pm 1.04$ $\mathrm{m} / \mathrm{s}$, the difference statistic was $\mathrm{p}=0.0008$. For the evaluation of the acceleration the straight hit, the following results are shown, $12.3 \pm 5.41 \mathrm{~m} / \mathrm{s}^{2}$, while the bandal chagi kick had an average result of $74 \pm 37.85 \mathrm{~m} / \mathrm{s} 2$, the statistical difference was of $\mathrm{p}=0.00033$ using a significance level of $\mathrm{p}=0.05$ for the measurements.

Keywords: boxing; Tae Kwon Do; attack strikes; combat sport; acceleration.

\section{INTRODUCCIÓN}

La gran mayoría de culturas han tenido una disciplina de combate propia y autóctona, que forma parte de su folklore y costumbres; ejemplo de ello, son el Sumo, en Japón; el Taekwondo, en Corea; Movy Thay, en Tailandia; el Kalí, en Filipinas y las luchadas, en Islas Canarias (Sánchez, 1999). Como se puede observar, los deportes de combate hacen parte del legado cultural y tienen su mayor expresión durante las competencias nacionales e internacionales, permitiendo, con ello, medir la evolución y el potencial deportivo, así como, la inversión de cada Nación.

Para que un deporte manifieste desarrollo y evolución y le permita ser representativo, a nivel internacional y hacer parte de las contiendas más importantes del planeta (Juegos Olímpicos), se requiere el apoyo de diferentes áreas interdisciplinarias, como son el entrenamiento, la medicina, la nutrición, la fisioterapia y la psicología. Dentro de los componentes del entrenamiento cabe destacar la función que cumple la biomecánica deportiva, que permite la caracterización técnica, por medio de implementos tecnológicos, que favorecen el análisis del deporte, desde componentes cinéticos y cinemáticos. (Aguado Jódar, 1993).

Los deportes objeto de análisis en el presente estudio son el boxeo y el taekwondo. El primero de ellos, ingresó al programa olímpico desde 1904 y se mantiene hasta la fecha; el segundo, a pesar de su ingreso a la palestra olímpica, en el 2000, ya era practicado en los cinco continentes, lo que equipara en desarrollo a estas dos disciplinas de combate (González Núñez \& Leyva Rodríguez, 2006). En Colombia, tanto el boxeo como el taekwondo, ofrecen un número importante de medallas en las contiendas nacionales e internacionales. Por lo anterior, los pugilistas y los taekwondocas pertenecen a un grupo de disciplinas deportivas de combate que se están estudiando de manera específica, con el fin de mejorar los factores físicos, técnicos, tácticos y estratégicos. Las variables más utilizadas desde el concepto biomecánico es el análisis de carácter cinemático y cinético (García Hernández et al. 2018)

Para el desarrollo de un estudio en el boxeo y en el teakwondo, se requiere caracterizar técnicamente los deportes. Los aspectos generales de las técnicas del boxeo están constituidos por un conjunto de métodos que, al ser bien utilizadas, conllevarán a la consecución de logros, ya que al pugilista le permiten resolver las dificultades que se desarrollan durante el combate (Degtiariov, 1983). Las variantes, los recursos, las maneras en que se ejecutan, el número total y la frecuencia de aplicaciones, dependerán de la velocidad con que se desarrollan las técnicas de ataque y defensa; por lo general, las técnicas de ataque se producen más intensamente que las de defensa.

Los recursos técnicos y sus variantes hacen parte de las características del boxeo. Las técnicas, se diferencian por los patrones motrices, las características cinemáticas, las dinámicas y los resultados de movimiento (Castarlenas \& Planas, 1997).

Los golpes en el boxeo constituyen el medio fundamental de ataque, que se realizan con la sección distal de las extremidades superiores (manos en forma de puños). Los golpes técnicos son: recto, gancho y cruzados. Se caracterizan por tener precisión, regularidad, contundencia y velocidad. Permite que los árbitros jueces evalúen todos los componentes mencionados y emitan su juicio del combate (Machuat Santana et al. 2017).

Por otro lado, el taekwondo es un arte marcial de origen coreano, que traduce "Camino del pie y la mano". Es una disciplina de combate, que se destaca por predominar los golpes con el pie (70\%), frente al golpe de las manos (30\%) (Pinzón Duque \& Trujillo Henao, 2002). En la tabla 1, se pueden observar las características técnicas básicas del taekwondo.

Los golpes del taekwondo proporcionan puntos en sistema electrónico de juzgamiento, siempre y cuando se realicen con el puño y el pie. Las zonas permitidas para puntuar en el tronco están cubiertas por el chaleco electrónico, que posee sensores de presión en la parte anterior y lateral, no posterior. También puntúan los golpes en el casco; no obstante, únicamente se permite la técnica de ataque con el pie para ello (Federación Colombiana de Taekwondo, 2018).

La patada Bandal chagui es uno de los gestos técnicos más usados entre todas las técnicas de ataque durante la competencia. Su ejecución comprende las siguientes fases: pierna dominante atrás, giro de cadera, flexión de la pierna y empeine; cerca al sensor de presión del chaleco, se realiza una extensión de tobillos y pierna, con el objetivo de impactar y producir la presión necesaria, para conseguir puntos durante el combate (De La Fuente García \& Castejón Oliva, 2016). Debido a la potencia y la velocidad que adquiere el gesto técnico de la patada Bandal Chagi, ha sido objeto de diversos estudios (Torres Ruiz et al. 2014), indicando la velocidad promedio de $8,55 \mathrm{~m} / \mathrm{s}$ por patada. Estos resultados, en conjunto con los análisis biomecánicos, permiten controlar y proyectar las 
Tabla 1. Clasificación de las técnicas del boxeo y el taekwondo (De La Fuente García \& Castejón Oliva, 2016).

\begin{tabular}{|c|c|c|c|}
\hline \multirow{15}{*}{$\begin{array}{l}\text { Técnicas } \\
\text { del boxeo }\end{array}$} & \multicolumn{2}{|c|}{ Tipos } & Modalidades \\
\hline & \multirow{3}{*}{\multicolumn{2}{|c|}{ Guardia posición combativa }} & Alta \\
\hline & & & Media \\
\hline & & & Baja \\
\hline & \multirow{5}{*}{\multicolumn{2}{|c|}{ Desplazamientos }} & Paso plano \\
\hline & & & Paso salto \\
\hline & & & Péndulo \\
\hline & & & Diagonales \\
\hline & & & Giros \\
\hline & \multirow{3}{*}{ Golpes } & Ataque & Rectos \\
\hline & & \multirow{2}{*}{ Contraataque } & Ganchos \\
\hline & & & Cruzados \\
\hline & \multirow{3}{*}{ Defensas } & Brazos & Izquierda \\
\hline & & Tronco & \multirow{2}{*}{ Derecha } \\
\hline & & Piernas & \\
\hline \multirow{23}{*}{$\begin{array}{c}\text { Técnicas } \\
\text { del } \\
\text { taekwondo }\end{array}$} & & & Modalidades \\
\hline & \multirow{3}{*}{\multicolumn{2}{|c|}{ Posición Combate }} & Alta \\
\hline & & & Media \\
\hline & & & Baja \\
\hline & \multirow{6}{*}{\multicolumn{2}{|c|}{ Desplazamientos }} & Adelante con cruce \\
\hline & & & Con cambio de posición \\
\hline & & & Adelante con desplazamiento de rodilla adelante \\
\hline & & & Desplazamiento con deslizamiento adelante \\
\hline & & & Desplazamiento hacia atrás con paso por adelante \\
\hline & & & Desplazamiento hacia adelante con giro \\
\hline & \multirow{3}{*}{\multicolumn{2}{|c|}{ Bloqueos }} & Con un brazo \\
\hline & & & Con dos brazos \\
\hline & & & Con brazo adelante y atrás \\
\hline & \multirow{10}{*}{ Golpes } & Puño & Rectos \\
\hline & & \multirow{9}{*}{ Patada } & Miro chagui \\
\hline & & & Bandal chagui \\
\hline & & & Tui chagui \\
\hline & & & Yop chagui \\
\hline & & & Dolyo chagui \\
\hline & & & Naryochagui \\
\hline & & & Furyo chagui \\
\hline & & & Bakat chagui \\
\hline & & & An chagui \\
\hline
\end{tabular}

cargas de entrenamiento, que repercutirá en mejores resultados, durante los encuentro deportivos (Vieten et al. 2007).

Las investigaciones de biomecánica en deportes de combate descomponen los movimientos desde factores cualitativos, cuantitativos, según las características que lo componen y las fases en que los pueden dividir (Tabla 2), con lo cual, se permite generar modelos técnico-deportivos, que analizan y proyectan las correctas ejecuciones. La cinemática trabaja por medio de coordenadas, ángulos, desplazamientos, velocidades y aceleraciones, entre otras, mientras que la cinética estudia las fuerzas que provocan el movimiento (Loachamin Aldaz et al. 2017). Si se tiene en cuenta que la generación de movimiento está supeditada al comportamiento motor, la evaluación y el control deportivo toman un papel determinante en la consecución de altos logros; no obstante, en la actualidad, algunos entrenadores aún se basan en controles de carácter observacional, que no requiere implementos de medición; son predominantemente cualitativos y se fundamenta en el conocimiento técnico (Berengüí et al. 2011). La posibilidad de incurrir en errores de apreciación aumenta, si se tiene en cuenta que un gesto técnico requiere de múltiples aproximaciones desde las ciencias del deporte, para entender el mismo. Menescardi et al. (2012) indican que cada acción técnica en los deportes de combate es un proceso sistemático, por tal razón, existe, entre las diferentes fases, una interdependencia. Si en el acumulado de este proceso cambia una de las fases, el cambio no solamente afecta a 
esa fase, sino que se transmite también a las demás. Por tal motivo, para futuras investigaciones, se proponen métodos de carácter cuantitativo, que estudien, desde la cinética y la cinemática, los patrones técnicos que requieren las disciplinas de combate, para afrontar, de manera adecuada, la preparación, la aproximación y la consecución de logros deportivos (Suárez, 2009; Aguado Jódar, 1993).

Tabla 2. Características del movimiento.
Para los deportes de combate, se especializan los segmentos corporales para conseguir la máxima eficiencia y eficacia durante las contiendas deportivas. Actualmente, en el ámbito deportivo, no se tiene información concluyente sobre la simetría o asimetría funcional de los golpes de ataque boxeo y el taekwondo, por lo cual, la presente investigación será un aporte al proceso de evaluación y de comparación cinemática de las técnicas de golpe Recto, en Boxeo y Bandal Chagui, en Taekwondo (Torres Ruiz et al. 2008).

\begin{tabular}{|c|c|c|c|c|c|c|}
\hline \multicolumn{3}{|c|}{ CUALITATIVO } & \multicolumn{4}{|c|}{ CUANTITATIVO } \\
\hline Dinámicos & Figurativos & Psicovolitivos & Dinámicos & Traslaciones & Rotacional & Cinemático \\
\hline Transición & Armonía & Anticipación & $\begin{array}{c}\text { Momento de } \\
\text { inercia }\end{array}$ & Espacio & Ángulo & Inercia \\
\hline Elasticidad & Amplitud & & $\begin{array}{c}\text { Momento de } \\
\text { fuerza }\end{array}$ & Velocidad & $\begin{array}{l}\text { Velocidad } \\
\text { angular }\end{array}$ & Velocidad \\
\hline Rapidez & Precisión & & $\begin{array}{l}\text { Momento } \\
\text { angular }\end{array}$ & Aceleración & $\begin{array}{c}\text { Aceleración } \\
\text { angular }\end{array}$ & Aceleración \\
\hline Intensidad & Constancia & & & & & \\
\hline Fluidez & & & & & & \\
\hline Ritmo & & & & & & \\
\hline
\end{tabular}

Adaptado de Bäumler \& Schneider (1989).

\section{MATERIALES Y MÉTODOS}

El presente estudio es observacional, de corte transversal y descriptivo (Gratton \& Jones, 2010). La muestra estuvo constituida por ocho boxeadores masculinos de Bogotá, con edades de $28,13 \pm 2,10$ años, masa corporal de $60,50 \pm 10,13 \mathrm{~kg}$, estatura $171 \pm 0,10 \mathrm{~cm}$ y ocho taekwondocas masculinos de Bogotá, con

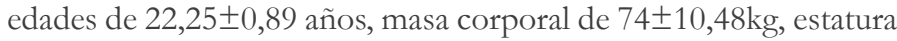
$1,79 \pm 0,09 \mathrm{~cm}$. Se capturó y se comparó por medio videográfico las velocidades y aceleraciones de los golpes Recto, en Boxeo y Bandal Chagui, en Taekwondo.

Las variables biomecánicas de velocidad y de aceleración de los golpes principales, se encuentran reportadas en la tabla 3.

Tabla 3. Datos generales de la muestra.

\begin{tabular}{|c|c|c|c|c|c|c|c|c|c|c|c|c|c|c|}
\hline \multirow{3}{*}{\begin{tabular}{|l} 
\\
Edad \\
\end{tabular}} & \multicolumn{7}{|c|}{ Boxeo Golpe Recto } & \multicolumn{7}{|c|}{ Taekwondo Golpe Bandal Chagui } \\
\hline & \multirow{2}{*}{$\begin{array}{l}\mathbf{n} \\
8 \\
\end{array}$} & \multicolumn{3}{|c|}{ Media \pm ds } & \multirow{2}{*}{$\begin{array}{c}\text { Mín } \\
25\end{array}$} & \multirow{2}{*}{$\begin{array}{c}\text { Máx } \\
31 \\
\end{array}$} & \multirow{2}{*}{$\begin{array}{l}\text { Coef. } \\
\text { Var. }\end{array}$} & \multirow{2}{*}{$\begin{array}{l}\mathbf{N} \\
8 \\
\end{array}$} & \multicolumn{3}{|c|}{ Media \pm ds } & \multirow{2}{*}{$\begin{array}{c}\text { Mín } \\
21 \\
\end{array}$} & \multirow{2}{*}{$\begin{array}{c}\text { Máx } \\
23 \\
\end{array}$} & \multirow{2}{*}{$\begin{array}{l}\text { Coef. } \\
\text { Var. }\end{array}$} \\
\hline & & 28,1 & \pm & 2,1 & & & & & 22,25 & \pm & 0,89 & & & \\
\hline Masa Corporal kg & 8 & 60,5 & \pm & 10,7 & 48 & 81 & & 8 & 74 & \pm & 10,78 & 58 & 88 & \\
\hline Estatura m & 8 & 1,71 & \pm & 0,1 & 1,59 & 1,92 & & 8 & 1,79 & \pm & 0,09 & 1,63 & 190 & \\
\hline Velocidad m/s & 8 & 4,17 & \pm & 1,35 & 2,5 & 7 & 32,37 & 8 & 11,75 & \pm & 1,04 & 11 & 14 & 8,85 \\
\hline Aceleración m/s ${ }^{2}$ & 8 & 12,3 & \pm & 5,41 & 4,43 & 21 & 43,98 & 8 & 74 & \pm & 37,85 & 14 & 149 & 51,15 \\
\hline
\end{tabular}

$\mathrm{n}=$ muestra / ds = Desviación estándar / Mín= Mínimo / Máx= Máximo. Coef. Var= Coeficiente de variación. 
La investigación, se rigió bajo las reglas establecidas por el Ministerio de Salud, para trabajos con seres humanos, Resolución No. 008430 de 1993 (Minsalud, 1993).

Criterios de inclusión: Boxeadores y taekwondocas que hicieran parte de las ligas nacionales de Bogotá, Colombia y que hubiesen tenido participación en competencias nacionales. Las pruebas, se llevaron a cabo en las instalaciones de las ligas de Taekwondo y Boxeo de Bogotá. Las evaluaciones fueron realizadas en una sesión y todos los deportistas se encontraban en la etapa inicial de preparación general.

Materiales y protocolo de medición:

1. Captura de las imágenes:

a) Para la captura de imágenes, se utilizó una cámara de video de alta velocidad de 60 cuadros por segundo, formato de norma NTSC, Sony HDR-XR100, alta definición HD y un trípode.

b) Se utilizó un sistema de referencia cubo de seis lados, medida de referencia, con la que el sistema compara las dimensiones para hacer los cálculos.

2. Planimetría de la ubicación de las cámaras y el sistema de referencia:

a) Digitalización de las imágenes, con la ayuda del sistema de análisis de movimiento HUMAN Ehuman Versión 5.0.

b) Tratamiento matemático de las imágenes, con el sistema de análisis de movimiento, HUMAN Ehuman Versión 5.0.

3. Tratamiento de las filmaciones y de los resultados:

a) Se utilizó un computador procesador Intel Core TM Dos Duo, RAM 2 Gb, sistema 32 bk. tarjeta de video y el software HU-M-AN Ehuman Versión 5.0.

b) Seefectuaron dos tratamientos estadísticos para determinar el nivel de significancia de los datos relacionados. Una prueba de Mann Whitney U. Test, debido al tamaño de la muestra y a la no parametricidad de los datos. Por otro lado, se realizó un coeficiente de variación para las velocidades y las aceleraciones por disciplina deportiva y una comparación porcentual, que determinó la diferencia entre los valores de las ejecuciones técnicas.

\section{RESULTADOS Y DISCUSIÓN}

Los datos generales de la población evaluada, la velocidad y la aceleración de los golpes rectos, en Boxeo y Bandal chagui, en Taekwondo, se encuentran reportados en la tabla 3. La dispersión en las edades de los boxeadores fue mayor que la de los taekwondocas, debido a que, la primera muestra, estuvo constituida por más de un rango etario, contrario a los taekwondocas, que únicamente fueron evaluados aquellos deportistas que fuesen menores a 23 años.

El coeficiente de variación intragrupal (Boxeo-Taekwondo) de la velocidad y la aceleración (Tabla 3), indica una mayor dispersión para los deportistas de boxeo (32,37\%), comparado con la de taekwondo (8,85\%) y se debe, a que la muestra de boxeo estuvo constituida por más de seis categorías de peso, mientras que la de los taekwondocas tuvo cuatro categorías de peso, entre los 8 evaluados. Teóricamente, la evidencia científica indica que hay una relación inversamente proporcional entre la masa corporal y la velocidad del golpe, ya que las divisiones de menor masa corporal reportan una mayor velocidad frente a las divisiones superiores (Nakano et al. 2014).

En la tabla 3, también se observan los resultados de velocidad y de aceleración. La diferencia entre velocidades de los golpes mostró que la patada de bandal chagui (taekwondo) es un 64,51\% más veloz que el golpe recto en boxeo. Los resultados reportados concuerdan con los expuestos por Sánchez (1999), quien indicó velocidades en el golpe de boxeo de $4-5 \mathrm{~m} / \mathrm{s}$ y en taekwondo, de $11,7 \mathrm{~m} / \mathrm{s}$, en promedio. Torres Ruiz et al. (2014) evaluaron la cinemática de la patada bandal chagui y reportaron una velocidad promedio de 8,55 $\mathrm{m} / \mathrm{s}$, semejantes a lo expuesto en el presente estudio (Tabla 3).

Consecuente con lo anterior, la patada bandal chagui (Figura 1) presenta una 83,38\% más de aceleración que el golpe recto en boxeo (Figura 2). Esta diferencia, se debe a dos factores: la cadena cinemática de la patada en taekwondo tiene una mayor amplitud de movimiento, mientras que, en el golpe recto en el boxeo, es más reducida (Figura 3). Según Donskoi \& Zatsiorski (1988), la ejecución de la patada en taekwondo se apoya en grandes grupos musculares del tren inferior y la zona lumbar, que participan en la rotación del tronco, desarrollando una cadena cinemática, que finaliza con el impacto en el adversario. El gesto técnico de la patada bandal chagui incluye una proyección de peso corporal, aproximadamente de un 19\%, lo que implica una mayor aceleración y velocidad, mientras que, en el boxeo, solo se implica el 5\% del peso total del pugilista (Degtiariov, 1983). Además, se deberá tener en cuenta que la masa impactante es inversamente proporcional a la velocidad; al aumentar la masa de choque disminuye la velocidad de desplazamiento y viceversa. En un contexto de divisiones de peso es relevante, ya que, con el aumento de las categorías, el control motor de la cadena cinemática y su fluidez, tienden a variar frente a un patrón ideal.

Finalmente, en la tabla 4, se presentan los resultados de la comparación con el test Mann Whitney U. Test, donde se evaluó la velocidad entre los dos golpes (recto y bandal chagui), arrojando una diferencia significativa bilateral de $\mathrm{p}=0.0008$, con un grado de significancia de $\mathrm{p}<0,05$; se puede aseverar, que hay una asimetría de la velocidad entre los golpes. Para el caso de la aceleración, nuevamente se encontró la misma asimetría, reportando un valor de $\mathrm{p}=0,0033$, con un grado de significancia de $\mathrm{p}<0,05$, lo que implica que, tanto la velocidad como la aceleración, presentan diferencias significativas entre los dos gestos técnicos evaluados.

El coeficiente de dispersión entre los grupos superó el 5\% para las dos muestras; no obstante, fue mayor para los pugilistas, debido a la variedad de categorías de peso que se evaluaron. 


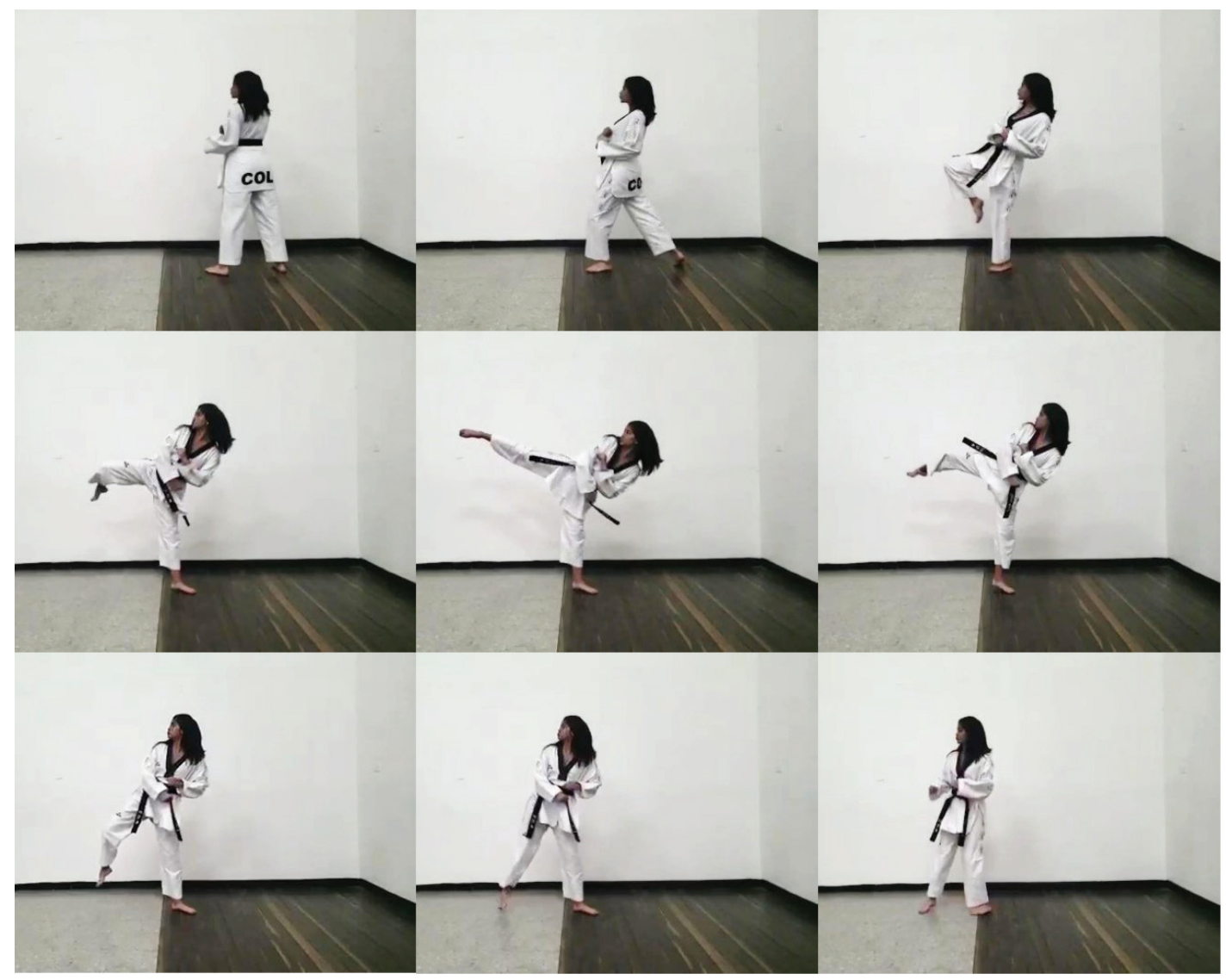

Figura 1. Patada Bandal Chagui en Taekwondo.

Del presente estudio se puede concluir que:

1. La patada bandal chagui en el taekwondo es un $64 \%$ más veloz que el golpe recto en el boxeo.

2. La patada bandal chagui presenta una $83,38 \%$ más de aceleración frente al golpe recto en boxeo.

3. La patada bandal chagui incluye una proyección de peso corporal mayor (19\%), frente al golpe recto, lo que implica una mayor aceleración y velocidad, a consecuencia de los rangos articulares ampliados.

Se recomienda, para futuros estudios, la utilización de dinamometría, para determinar la contundencia de los golpes, correlacionándolos con la velocidad y la aceleración, así como tener en cuenta la misma cantidad de deportistas, por división de peso.
Según las pruebas estadísticas hay una asimetría entre los gestos técnicos evaluados, por lo cual, se propone, para futuros estudios, realizar evaluaciones de las acciones técnicas, únicamente de tren inferior o superior, para determinar las variaciones entre los mismos.

Agradecimiento: Se da agradecimiento especial al estudiante David Peña, de la Universidad de Ciencias Aplicadas y Ambientales U.D.C.A, Facultad de Ciencias del Deporte, por su apoyo durante la ejecución del estudio. Conflicto de intereses: El manuscrito fue preparado y revisado con la participación de todos los autores, quienes declaramos que no existe conflicto de intereses, que ponga en riesgo la validez de los resultados presentados. Financiación: La presente investigación fue financiada por la Universidad de Ciencias Aplicadas y Ambientales U.D.C.A. 


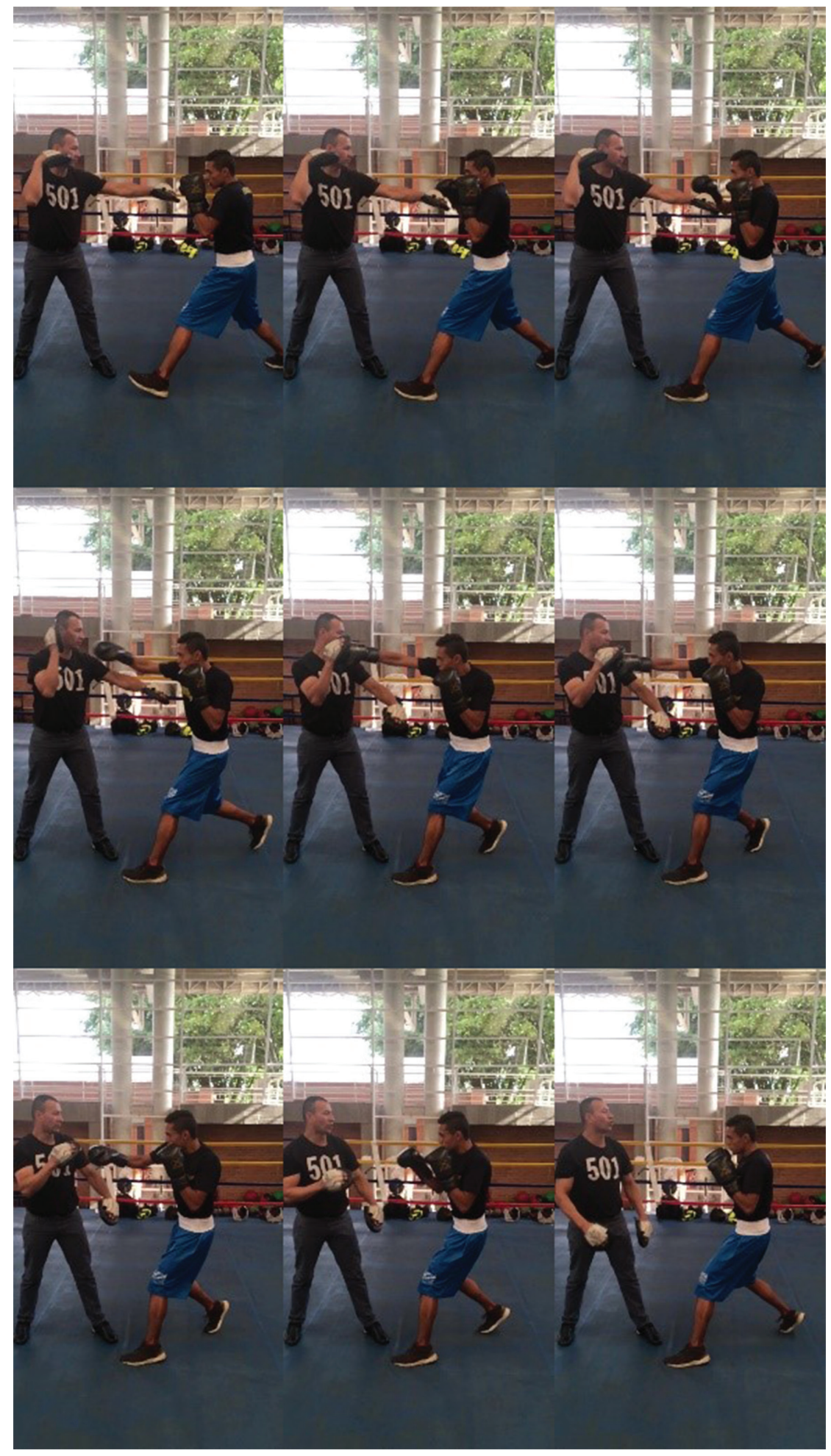

Figura 2. Golpe Recto en Boxeo. 

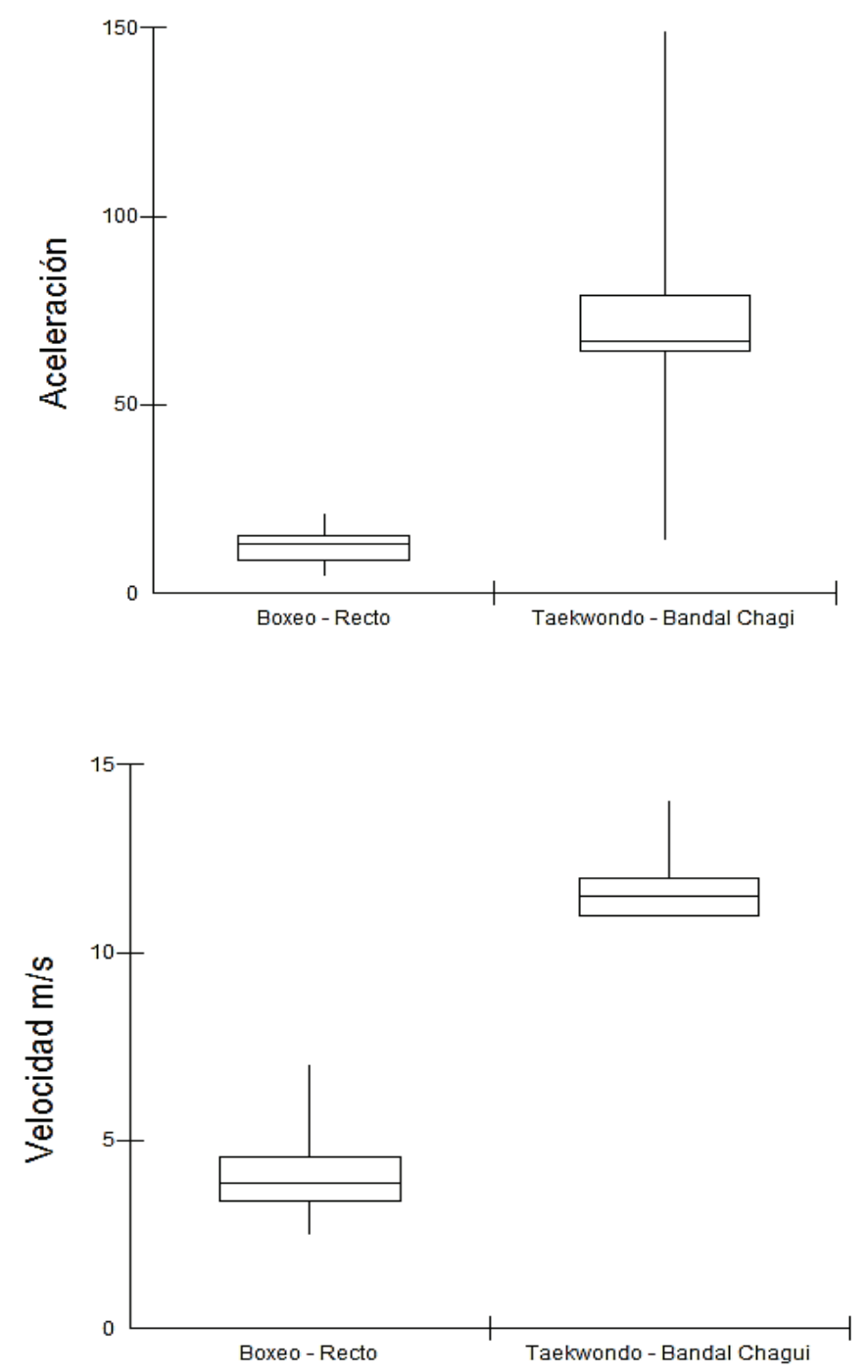

Figura 3. Velocidad y aceleración en los golpes, Recto en Boxeo y Bandal Chagui, en Taekwondo.

Tabla 4. Mann Whitney U. Test evaluando velocidad y aceleración en los golpes Recto, en Boxeo y Bandal Chagui, en Taekwondo.

\begin{tabular}{|l|c|c|c|c|}
\hline \multicolumn{1}{|c|}{ Resultado } & $\begin{array}{c}\text { Velocidad } \\
\text { Boxeo }\end{array}$ & $\begin{array}{c}\text { Velocidad } \\
\text { Taekwondo }\end{array}$ & $\begin{array}{c}\text { Aceleración } \\
\text { Boxeo }\end{array}$ & $\begin{array}{c}\text { Aceleración } \\
\text { Taekwondo }\end{array}$ \\
\hline $\begin{array}{l}\text { Tamaño de la } \\
\text { Muestra }\end{array}$ & 8 & 8 & 8 & 8 \\
\hline $\begin{array}{l}\text { Suma de los Puestos } \\
\text { Ri }\end{array}$ & 36,0 & 100,0 & 40,0 & 96,0 \\
\hline Mediana & 3,89 & 11,50 & 13,06 & 67,00 \\
\hline $\mathrm{U}=$ & 0,00 & & 4,00 & \\
\hline ZU $=$ & 33607 & & 29,406 & \\
\hline p-valor unilateral $=$ & 0,0004 & & 0,0016 & \\
\hline p-valor bilateral $=$ & $0,0008^{*}$ & & $0,0033 *$ & \\
\hline
\end{tabular}

* Diferencia significativa entre la velocidad de los dos tipos de golpe $\mathrm{p}<0,05$ 


\section{REFERENCIAS}

1. AGUADO JÓDAR, X. 1993. Eficiencia y técnica deportiva. Análisis del movimiento humano. Barcelona, España: Publicaciones INDE. Cap. 4. p.58-73.

2. BÄUMLER, G.; SCHNEIDER, K. 1989. Biomecánica deportiva: fundamentos para el estudio y la práctica. Barcelona: Martínez Roca. 141p.

3. BERENGÜÍ GIL, R.; LÓPEZ GULLÓN, J.M.; GARCÉS DE LOS FAYOS RUIZ, E.J.; ALMARCHA TERUEL, J. 2011. Factores psicológicos y lesiones deportivas en lucha olímpica y taekwondo. E-balonmano.com: Revista de Ciencias del Deporte. 7:91-98.

4. CASTARLENAS, J.L.; PLANAS, A. 1997. Estudio de la estructura temporal del combate de judo. Apunts: Educación Física y Deportes. 47:32-39.

5. DEGTIARIOV, I.P. 1983. Boxeo. Ed. Raduga. Raduga. Rusia. p.257-281.

6. DONSKOI, D.; ZATSIORSKI, V. 1988. Biomecánica de los Ejercicios Físicos. Editorial Raduga, Moscú, Rusia. p.180192.

7. DE LA FUENTE GARCÍA, A.M.; CASTEJÓN OLIVA, F.J. 2016. Análisis del combate en taekwondo. Categorías para la evaluación de las acciones tácticas. Estudio preliminar. Cultura, ciencia y deporte: revista de ciencias de la actividad física y del deporte de la Universidad Católica de San Antonio. (España). 32:157-170. http://dx.doi. org/10.12800/ccd.v11i32.715

8. FEDERACIÓN COLOMBIANADETAEKWONDO. 2018. Reglamento e interpretación de arbitraje en combate de taekwondo. Disponible desde Internet en: http:/ / mastkd. com/wp-content/uploads/2018/09/Kyorugi-01_ ES_REGLAMENTO-E-INTERPRETACION-DEARBITRAJE-EN-COMBATE-DE-TAEKWONDOW'T.pdf (con acceso 18/02/19).

9. GARCÍA HERNÁNDEZ, J.M.; MACHUAT SANTANA, G.; FERNÁNDEZ JIMENEZ, B.; TOLEDO DÍAZ, P.O. 2018. La preparación técnico-táctica de los boxeadores espirituanos. Podium. Rev. Ciencia y Tecnología en la Cultura Física. 13:150-159.

10. GONZÁLEZ NÚÑEZ, A.M.; LEYVA RODRÍGUEZ, J. 2006. Planificación del entrenamiento deportivo en el taekwondo atendiendo a las zonas de producción de energía en las diferentes direcciones de la preparación. Efdeportes. Buenos Aires. 10(93).
11. GRATTON, C.H.; JONES, J. 2010. Research Methods for Sport Studies. Taylor \& Francis. 304p.

12. LOACHAMIN ALDAZ, E.M.; MENA PILA, F.M.; DURÁN PORTILLA, E.E.; MAQUEIRA CARABALLO, G. DE LA. C.; CHÁVEZ CEVALLOS, E. 2017. Diferencias biomecánicas en la patada Ap Chagüi entre taekwondocas de cinturón blanco y negro. Rev. Cubana Investigaciones Biomédicas.36(2):159-168.

13. MACHUAT SANTANA, G.; RAMOS QUIAN, Y.; RAMOS GARCÍA, L. 2017. Ejercicios técnicos para mejorar la efectividad en la ejecución de los golpes rectos a la cara y al tronco de los boxeadores de la categoría 11 y 12 años. PODIUM - Rev. Ciencia y Tecnología en la Cultura Física. 12(3):178-188.

14. MENESCARDI, C.; BERMEJO, J.L.; HERRERO, C.; ESTEVAN, I.;LANDEO, R.;FALCO, C. 2012. Diferencias técnico-tácticas en taekwondistas universitarios según sexo y categoría de competición. Rev. Artes Marciales Asiáticas. 7(2):1-11. https://doi.org/10.18002/rama.v7i2.82

15. MINISTERIO DE SALUD - MINSALUD. RESOLUCIÓN No 008430 DE 1993 (4 DE OCTUBRE DE 1993) Por la cual se establecen las normas científicas, técnicas y administrativas para la investigación en salud. Disponible desde Internet en: https://www.buenastareas.com/ ensayos/Resolucion-8430-De-1993/1497509.html

16. NAKANO, G.; IINO, Y.; IMURA, A.; KOJIMA, T. 2014. Transfer of momentum from different arm segments to a light movable target during a straight punch thrown by expert boxers. J. Sports Sciences 32(6). https://doi.org/1 $0.1080 / 02640414.2013 .843014$

17. PINZÓN DUQUE, O.A.; TRUJILLO HENAO, S.E. 2002 Análisis de la patada Dollyo Chagui en Taekwondo. Rev. Med Risaralda. 8(2):1-8.

18. SÁNCHEZ, R.;D.A. 1999. Algunas características cuantitativas de los gestos de ataque del boxeo; rectos, ganchos y cruzados, de un atleta con ayuda de un sistema de análisis de movimiento en dos dimensiones con criterios cinemáticos. Acción. Revista Cubana de la Cultura física. 1:65-73.

19. SUÁREZ, G.R. 2009. Biomecánica Deportiva y Control del Entrenamiento. Medellín: Funánbulos Editores. p.65-83.

20. TORRES RUIZ, A.; MANRIQUE, J.; ARDILA S. 2014. Caracterización Digital de la Biomecánica del Gesto de la Patada en la Disciplina de Taekondo. Memorias XXIV Congresso Brasileiro de Engenharia Biomédica - CBEB. p.1058-1061. 
21. TORRES RUIZ, A.; CARRILLO RIVERA, A.R.; GONZÁLEZ BARRERA, C.A.; MURCIA TORRES, D.R. 2008. Caracterización digital de la biomecánica de los gestos deportivos para el boxeo. Entérese. (Colombia). 25:101-106.
22. VIETEN, M.; SCHOLZ, M.; KILANI, H.; KOHLOEFFEL, M. 2007. Reaction time in taekwondo. Proceedings of XXV. International Symposium on Biomechanics in Sports, Ouro Preto, Brazil, p.293-296. 